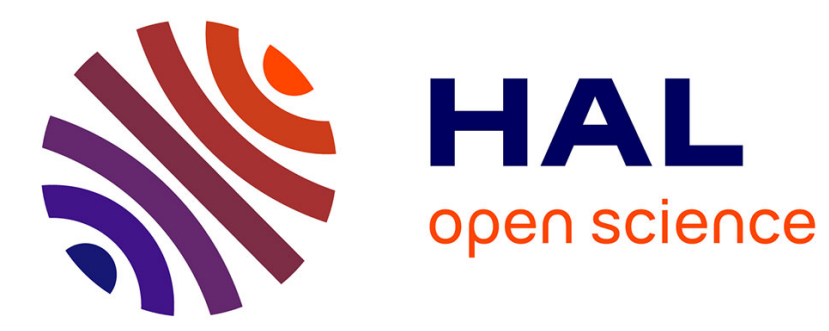

\title{
Détermination des contraintes résiduelles et de la microstructure intra-granulaire dans des films minces de W déposés par pulvérisation magnétron
}

\author{
V. Branger, N. Durand, A. Haghiri-Gosnet, K. Badawi, M. Ravet
}

\section{- To cite this version:}

V. Branger, N. Durand, A. Haghiri-Gosnet, K. Badawi, M. Ravet. Détermination des contraintes résiduelles et de la microstructure intra-granulaire dans des films minces de $\mathrm{W}$ déposés par pulvérisation magnétron. Journal de Physique III, 1997, 7 (6), pp.1247-1260. 10.1051/jp3:1997186 . jpa-00249645

\section{HAL Id: jpa-00249645 https://hal.science/jpa-00249645}

Submitted on 1 Jan 1997

HAL is a multi-disciplinary open access archive for the deposit and dissemination of scientific research documents, whether they are published or not. The documents may come from teaching and research institutions in France or abroad, or from public or private research centers.
L'archive ouverte pluridisciplinaire HAL, est destinée au dépôt et à la diffusion de documents scientifiques de niveau recherche, publiés ou non, émanant des établissements d'enseignement et de recherche français ou étrangers, des laboratoires publics ou privés. 


\title{
Détermination des contraintes résiduelles et de la microstructure intra-granulaire dans des films minces de $W$ déposés par pulvérisation magnétron
}

\author{
V. Branger $\left({ }^{1}\right)$, N. Durand $\left({ }^{1}\right)$, A.M. Haghiri-Gosnet $\left({ }^{2}\right)$, K.F. Badawi $\left({ }^{1, *}\right)$ et \\ M.F. Ravet ( $\left.{ }^{2}\right)$
}

$\left({ }^{1}\right)$ Laboratoire de Métallurgie Physique, $\left({ }^{*}\right)$, boulevard 3 Teléport 2, BP179, 86960 Futuroscope cedex, France

$\left({ }^{2}\right)$ Laboratoire de Microstructures et Micro-électronique, C.N R.S., 196 avenue H Ravera, 92225 Bagneux, France

(Reçu le 4 jullet 1996, révisé le 6 février 1997, accepté le 26 février 1997)

PACS.68. - Surfaces and Interfaces; thin films and whiskers

Résumé. - Cet article concerne l'étude des propriétés mécaniques et microstructurales de films minces de tungstène (700 nm sur Si (100)) élaborés par pulvérisation magnétron en fonction de la puissance R.F. Les contraintes totales présentes dans les films (déterminées par la méthode de la flèche) sont très fortement influencées par la valeur de la puissance R.F. Elles varient de $+1,2 \mathrm{GPa}$ à $-1,5 \mathrm{GPa}$ pour des puissances de $350 \mathrm{~W}$ à $400 \mathrm{~W}$ L'analyse par diffraction des rayons $\mathrm{X}$ a révélé la présence de phase $\beta$-W dans certains dépôts Pour ces films, nous avons détermıné les contraintes dans chaque phase par la méthode des $\sin ^{2} \psi$. Les mesures d'intensité intégrée des pics de diffraction nous ont permis d'évaluer la proportion des phases dans chaque film Nous avons vérifié le bon accord, en appliquant la règle des mélanges, entre les valeurs des contraintes totales et celles calculées à partir des résultats de diffraction $\mathrm{X}$. Nous avons montré que la présence de la phase $\beta$-W semble favorisée par un état de tension des films (jusqu'à $60 \%$ en volume pour des dépôts en tension de $+1,2 \mathrm{GPa}$ ) Le paramètre de maille libre de contrainte varie de façon spectaculaire. Il est très supérıeur à celui du $\mathrm{W}$ massif pour les dépôts en compression $(0,3173 \mathrm{~nm}$ au lieu de $0,3165 \mathrm{~nm})$, et légèrement inférieur pour ceux en tension $(0,3164 \mathrm{~nm})$.

\begin{abstract}
Intra-granular microstructure $\left(a_{0}\right)$ and stress of tungsten $\mathrm{rf}$ magnetron sputtered thin films $(<700 \mathrm{~nm}$ ) have been correlated for deposits belonging to the T zone of the Tornton's microstructural diagram. The stress values determined from the curvature method are compared with those calculated from the XRD method in each diffracting phase ( $\alpha-W$ and $\beta-W)$. The stress state is imposed by the rf magnetron sputtering poxer $(P)$ The films deposited with a high $P$ are in a large compression stress state (1n the $\alpha$-W phase) and own a free stress lattice parameter higher than the tungsten bulk one. We have quantitatively shown that the transition of the stress state from the compressive $(-1.7 \mathrm{GPa})$ to the tensile one $(0.9 \mathrm{GPa})$ was clearly explained by the intra-granular modification (diminution of the stress free lattice prarameter from $0.3164 \mathrm{~nm}$ ) From the XRD experiments, we have measured the coherently diffracting domains and the microdistorsions, which respectively decrease and increase with the generation of compressive stresses in the films. In addition, we have observed the dependence of the $\beta-\mathrm{W}$ phase with the tension in the films.
\end{abstract}

$\left.{ }^{*}\right)$ Auteur auquel doit être adressée la correspondance

(*) U R.A. C.N.R.S. 131

(C) Les Éditions de Physique 1997 


\section{Introduction}

Les contraintes intrinsèques présentes dans les films minces déposés sur un substrat modifient fortement leurs propriétés optiques, électriques ou encore magnétiques. Dans la plupart des applications, en particulier pour les dépôts de tungstène utilisés comme couche absorbante dans les masques à rayons $\mathrm{X}$ ou comme contact dans des transistors à hétéro-jonction, il est indispensable que les films ne soient soumis qu'à une contrainte très faible. Ceci explique l'intérêt porté à la compréhension de leur genèse lors du dépôt.

L'origine et la nature des contraintes intrinsèques dans les films minces, ont été très souvent reliées à la microstructure inter-granulaire des films. Dans des dépôts de tungstène déposés par pulvérisation magnétron, Hoffmann et al. [1] ainsi que Haghiri-Gosnet et al. [2] ont observé une très grande influence des paramètres de dépôts (puissance radiofréquence (rf) ou température du substrat pendant le dépôt) sur le signe et sur l'amplitude des contraintes résiduelles dans les films. En particulier ces études ont mis en évidence une inversion rapide du signe des contraintes dans un domaine de température ou de fréquence très restreint. Parallèlement, ces résultats ont été corrélés avec la morphologie des films déterminée par microscopie électronique à balayage. Thornton [3] a proposé une classification, en fonction des paramètres de dépôt, des différentes morphologies structurelles rencontrées. Il a relié ces différentes morphologies à une évolution qualitative des contraintes. Un paramètre essentiel est le rapport, température du substrat pendant le dépôt $\left(T_{\mathrm{s}}\right)$ sur température de fusion du matériau $\left(T_{\mathrm{m}}\right)$. Pour les faibles valeurs de ce rapport $\left(T_{\mathrm{s}}<0,15 T_{\mathrm{m}}\right.$, zone $\left.\mathrm{I}\right)$, la faible mobilité des atomes déposés conduit à une structure poreuse du film de type colonnaire et est très souvent associée à des contraintes de tension dans le film. Pour 0,3> $T_{\mathrm{s}} / T_{\mathrm{m}}>0,15$ (zone $\mathrm{T}$ ), le film devient dense avec une brusque variation de la contrainte interne.

Dans le but de compléter ces études à caractère inter-granulaire nous nous proposons d'étudier en détail la transition zone I-zone $\mathrm{T}$ du diagramme de Thornton, qui correspond au passage d'un état de tension vers état de compression. Notre but ici est également de relier l'état mécanique à l'état microstructural intra-granulaire de films minces de tungstène obtenus par pulvérisation magnétron dans des conditions expérimentales proches des études précédentes Nous avons utilisé deux méthodes de caractérisation : la méthode classique dite de la "flèche" (utilisée dans les études précédemment citées) et la diffraction des rayons $\mathrm{X}$. Cette dernière technique a pẹrmis une caractérisation complète des dépôts : analyse de la texture, identification et quantification des phases en présence, détermination des contraintes résiduelles, détermination du paramètre cristallin libre des contraintes, etc. Comme l'ont montré Noyan et Goldsmith $[4,5]$, l'association des résultats de ces deux méthodes complémentarres de détermination des contraintes résiduelles (flèche et " $\sin ^{2} \psi$ ") permet de distinguer la contribution des contraintes intra-granulaires de celle des contraintes inter-granulaires d'un film mince.

Dans cet article, après avoir présenté les différentes techniques de détermination des contraintes résiduelles ainsi que les conditions opératoires, nous exposerons les résultats de notre étude de la zone d'inversion du signe des contraintes. En premier lieu, nous comparerons et commenterons les résultats obtenus par les deux méthodes de détermination, et dans une seconde partie, nous mettrons en évidence une évolution de la microstructure intra-granulaire simultanément à l'inversion du signe des contraintes. Dans une dernière partie en nous inspirant des modèles phénoménologiques généralement admis pour la genèse des contraintes résiduelles dans les films minces déposés par pulvérisation, nous montrerons comment il est possible de relier quantitativement cette évolution microstructurale à l'apparition de contraintes de compression de grande amplitude 
1.1. MÉTHOdE DES " $\sin ^{2} \psi$ ". - Cette méthode, qui utilise la diffraction des rayons $\mathrm{X}$, consiste à effectuer une mesure de déformation $\varepsilon_{\phi \psi}$ et à remonter aux contraintes à partir des relations de l'élasticité linéaire. Dans cette méthode, la jauge de déformation utilisée est la distance interréticulaire $d_{h k l}$ d'une famille de plans cristallins $(h k l)$. La définition dite exacte ou rationnelle des déformations relie la variation de $d_{h k l}$ à la déformation $\varepsilon_{\phi \psi}$ de la façon suivante [6] :

$$
\varepsilon_{\phi \psi}=\ln \left(d_{h k l} / d_{0}\right)
$$

où $d_{0}$ est la distance interréticulaire en l'absence de contrainte, les angles $\phi$ et $\psi$ sont les angles classiques d'Euler. En utilisant la loi de Bragg, les déformations $\varepsilon_{\phi \psi}$ s'écrivent [7] :

$$
\varepsilon_{\phi \psi}=\ln \left(\sin \theta_{0} / \sin \theta_{\phi_{\psi}}\right) .
$$

Nous supposerons, par la suite que $\sigma_{33}=0$ (équilibre mécanique à la surface du film), que le film est soumis à un champ de contraintes isotrope dans son propre plan $\left(\sigma_{11}=\sigma_{22}=\sigma\right)$ et que les cisaillements sont négligeables. Ces hypothèses ont été vérifiées expérimentalement à plusieurs reprises $[8,9]$. En utilisant les relations de l'élasticité linéaire entre contraintes et déformations, dans le cas de films polycristallins et non texturés, on obtient la relation suivante [7] :

$$
\ln \left(1 / \sin \theta_{\psi}\right)=\left(1+\nu_{\mathrm{f}}\right) / E_{\mathrm{f}} \sigma \sin ^{2} \psi-2 \nu_{\mathrm{f}} / E_{\mathrm{f}} \sigma+\ln \left(1 / \sin \theta_{0}\right)
$$

où $E_{\mathrm{f}}$ et $\nu_{\mathrm{f}}$ sont respectivement le module d'Young et le coefficient de Poisson du film. Ln $\left(1 / \sin \theta_{\psi}\right)$ est une fonction linéaire de $\sin ^{2} \psi$ dont la pente permet la détermination de $\sigma$ et l'ordonnée à l'origine celle de $\sin \theta_{0}$. On remonte facilement au paramètre de maille libre de contraintes $a_{0}$ par l'intermédiaire de la loi de Bragg.

Cette donnée microstructurale $\left(a_{0}\right)$ est extrêmement importante pour l'étude de la structure intra-granulaire des films minces car elle reflète directement la présence et la nature de défauts ponctuels dans la phase diffractante. Cette méthode permet, de plus, la détermination sélective des contraintes dans plusieurs phases diffractantes d'un même film ; par exemple, dans chacun des constituants d'alliages métalliques polyphasés massifs $[10,11]$ ou encore dans la phase $\alpha$-W (cubique centrée) et dans la phase $\beta$-W (cubique A15) qui peuvent être présentes simultanément dans un dépôt de tungstène.

1.2. MÉTHODE DE LA FLÈCHE. — La méthode de la flèche est très majoritairement employée pour déterminer les contraintes résiduelles dans les films minces. Elle est rapide, simple de mise en œuvre, non destructive et applicable à tout matériau (cristallin ou amorphe). Quand un film adhérent à un substrat se trouve soumis à des contraintes biaxiales, il exerce des forces sur le substrat qui entraînent une courbure de ce dernier. Si son module d'Young est isotrope dans le plan du film, le substrat prend une forme sphérique. La relation entre la contrainte moyenne dans le film, $\sigma_{t}$, et le rayon de courbure du substrat, $R$, est donnée par la formule de Stoney [12] ; cette formule est modifiée par une éventuelle courbure du substrat avant le dépôt du film, de la façon suivante [13] :

$$
\sigma_{\mathrm{t}}=\left(E_{\mathrm{s}} / 1-\nu_{\mathrm{s}}\right)\left(H^{2} / 6 h\right)\left(1 / R_{\mathrm{f}}-1 / R_{\mathrm{l}}\right)
$$

où $E_{\mathrm{s}}$ et $\nu_{\mathrm{s}}$ sont respectivement le module d'Young et le coefficient de Poisson du substrat, $H$ l'épaisseur du substrat, $h$ celle du film. $R_{\mathrm{i}}$ et $R_{\mathrm{f}}$ sont les rayons de courbure du substrat avant et après dépôt du film.

La méthode de la flèche donne une valeur de contrainte que nous qualifierons de totale dans la suite de cet article. Elle représente une moyenne sur l'ensemble du film (toutes les phases, zones amorphes, joints de grains, amas de défauts...) à l'opposé de la valeur de contrainte 
(intra-granulaire) obtenue par diffraction des rayons $\mathrm{X}$ qui ne représente qu'une moyenne sur le volume diffractant de la phase étudiée.

On remarquera que la méthode de la flèche utilise le module d'Young et le coefficient de Poisson du substrat dont les valeurs sont parfaitement connues, alors que la méthode des " $\sin ^{2} \psi "$ nécessite l'utilisation des constantes élastiques du film. Celles-ci ne sont généralement pas connues précisément et seront supposées égales à celles du matériau massif dans la suite de cet article. Toutefois, la mise au point d'une mini-machine de traction est en cours pour déterminer les constantes élastiques des films minces et ainsi lever cette indétermination.

\section{Dispositif Expérimental}

Les conditions de dépôt des films de tungstène sont identiques à celles de la référence [8]. Les films, d'une épaisseur de $700 \mathrm{~nm}$ à $\pm 5 \%$, ont été déposés sur silicium monocristallin (100) poli optique (d'épaisseur $200 \mu \mathrm{m}$ et $600 \mu \mathrm{m}$ ) en utilisant un dispositif magnétron radio-fréquence (rf) de type Alcatel SCM 600. La puissance rf, fournie lors du dépôt, varie de $350 \mathrm{~W}$ à $400 \mathrm{~W}$ (avec une précision de $\pm 2 \mathrm{~W}$ ) ce qui correspond respectivement à des températures du substrat de 400 et 450 degrés Celsius. Avant dépôt, le vide dans l'enceinte est inférieur à $10^{-7}$ Torr. Après leur sortie de la chambre de dépôt, les échantillons sont conservés à température ambiante sous atmosphère d'argon afin d'éviter une importante adsorption d'oxygène à la surface du film.

Les mesures par diffraction des rayons $\mathrm{X}$ ont été réalisées au Laboratoire de Métallurgie Physique (Poitiers, France) où un générateur à anode tournante ( $5 \mathrm{~kW}$ ) équipé d'un monochromateur, délivre la raie $\mathrm{K} \alpha_{1}$ du cuivre $(\lambda=0,15406 \mathrm{~nm})$. Nous avons utilisé un diffractomètre deux cercles (géométrie $\theta / 2 \theta$ ), muni d'un détecteur linéaire. Ce dispositif, spécialement, adapté pour l'étude des films minces, est entièrement sous vide primaire. Ces mesures ont été confirmées au L.U.R.E. (Orsay, France), en utilisant le rayonnement synchrotron.

Expérimentalement, pour la détermination des contraintes résiduelles, nous avons choisi la raie de diffraction $(321)$ de la phase $\alpha-W\left(2 \theta \approx 131^{\circ}\right)$ et la raie $(611)$ de la phase $\beta$-W $\left(2 \theta \approx 141^{\circ}\right)$ pour 9 valeurs d'angle $\psi(0, \pm 10, \pm 30, \pm 45, \pm 55)$. La position des pics de diffraction est déterminée par la position de son barycentre, après soustraction du bruit de fond et correction L.P.A. (Lorentz-Polarisation-Absorption). La résolution de notre dispositif est de 0,05 en $2 \theta$. Les calculs d'incertitudes sur les valeurs des contraintes ont été réalisés selon la méthodologie proposée par Kalhoun et al. [14]. Les valeurs du module d'Young et du coefficient de Poisson pour le tungstène utilisés dans cet article sont respectivement $389 \mathrm{GPa}$ et 0,284.

Pour la détermination des microdéformations et de la taille des domaines cohérents de diffraction présents dans les films, nous avons employé la méthode dite de la largeur intégrale. Cette méthode a été largement traitée dans la littérature $[7,15,16]$ et employée plus récemment par Durand dans le cas de films minces d'or [17]. À partir de l'enregistrement de deux pics de diffraction d'une même famille cristallographique, pour deux ordres différents, cette méthode permet de déterminer les microdistortions présentes dans le dépôt et la taille des cristallites $\mathrm{D}$ dans la direction de croissance du film, en supposant gaussienne les différentes contributions à l'élargissement des pics de diffraction. Dans notre étude, nous avons utilisé les deux pics de diffraction (110) et (220) de la phase $\alpha$-W enregistrés en géométrie $\theta / 2 \theta$.

Pour déterminer les contraintes par la méthode de la flèche, il existe de nombreuses techniques permettant la mesure du rayon de courbure du substrat. Campbell [18] a fait une revue de celles qui sont généralement employées. Dans cette étude, nous avons utilisé un dispositif d'interférométrie Fizeau et un microprofilomètre mécanique de type DEKTACK IIA. Nous avons pris comme valeur du module biaxial du silicium : $E_{\mathrm{s}} /\left(1-\nu_{\mathrm{s}}\right)=180,5 \mathrm{GPa}[19]$. 


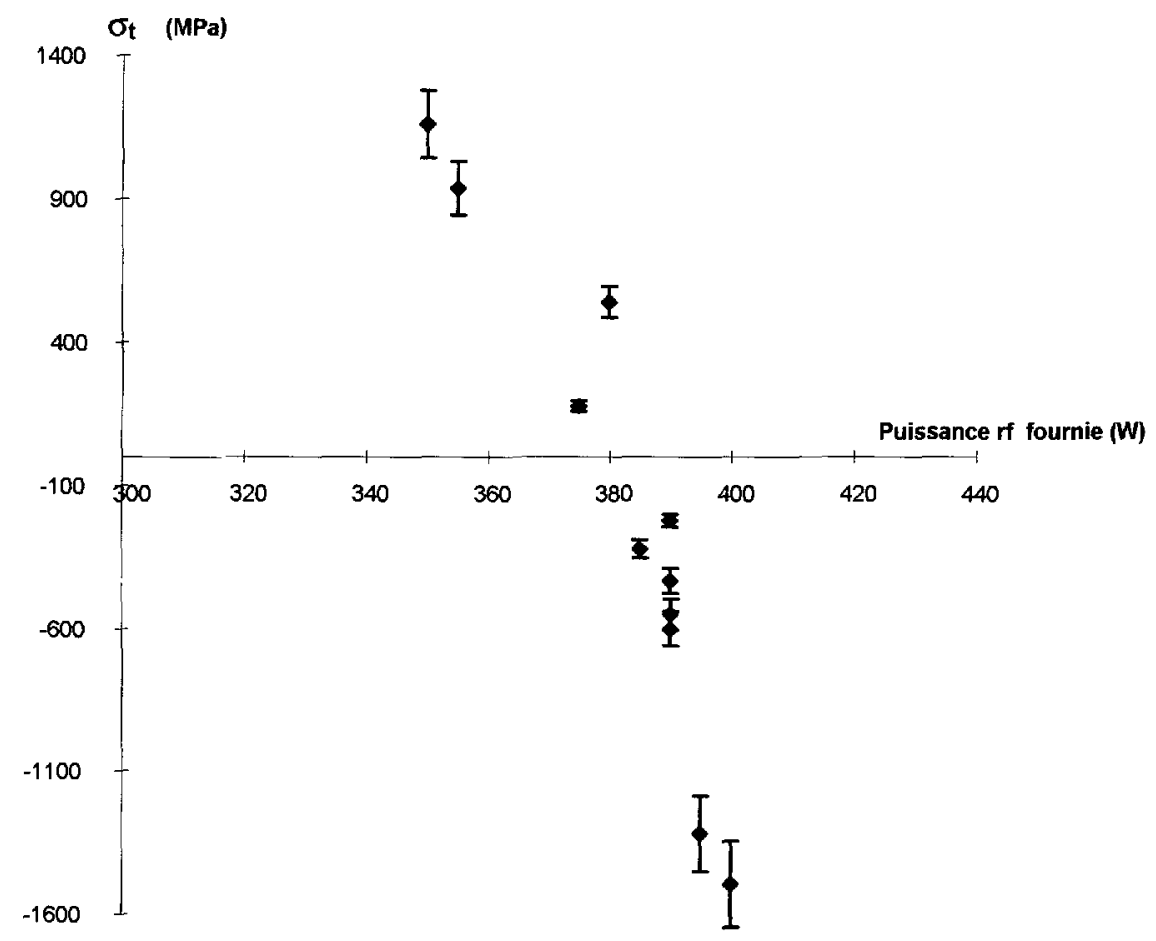

Fig. 1 - Contrainte totale dans le film en fonctıon de la puissance rf fournie lors du dépôt (W).

[Total stress versus rf magnetron sputtering power (W).]

Les valeurs des contraintes totales déterminées par les deux techniques de mesure de courbure (interférométrie et microprofilométrie mécanique), sont parfaitement cohérentes Les écarts observés sont toujours inférieurs aux incertitudes de mesure estimées à $\pm 10 \%$.

\section{Résultats et discussion}

3.1. Contraintes Résiduelles totales. - Les dépôts sont parfaitement adhérents aux substrats de Si et présentent une bonne tenue mécanique. Pour l'étude de la texture des films, nous avons réalisé pour chaque dépôt une "rocking curve" sur les pics de diffraction (110) de la phase $\alpha-W$. Cette analyse n'a révélé aucune direction privilégiée de croissance du film, quelque soit le dépôt et la phase analysée.

Sur la figure 1, nous avons représenté la contrainte totale des films en fonction de la puissance rf fournie lors du dépôt. Les contraintes varient d'une tension de 1,2 $\pm 0,2 \mathrm{GPa}$, pour une puissance de $350 \mathrm{~W}$, à une compression de $-1,5 \pm 0,2 \mathrm{GPa}$ pour une puissance de $400 \mathrm{~W}$. Cette inversion brutale du signe des contraintes a déjà été observée et très largement discutée dans une région de puissance rf similaire $[1,2,5]$.

Les contraintes thermiques sont le résultat du différentiel de dilatation thermique $(\Delta \alpha)$ entre le film et le substrat [9]. Pour un dépôt réalisé avec une puissance rf de $350 \mathrm{~W}$ (respectivement $400 \mathrm{~W}$ ), la température du substrat est de l'ordre de $400^{\circ} \mathrm{C}\left(450^{\circ} \mathrm{C}\right.$ ). Ainsi, en prenant $\Delta \alpha=2,2 \times 10^{-6} \mathrm{~K}^{-1}[19]$, on trouve une contrainte de $+0,4 \mathrm{GPa}(+0,5 \mathrm{GPa})$. En conséquence, même si l'on ne peut pas négliger leur présence, les contraintes thermiques ne peuvent pas 


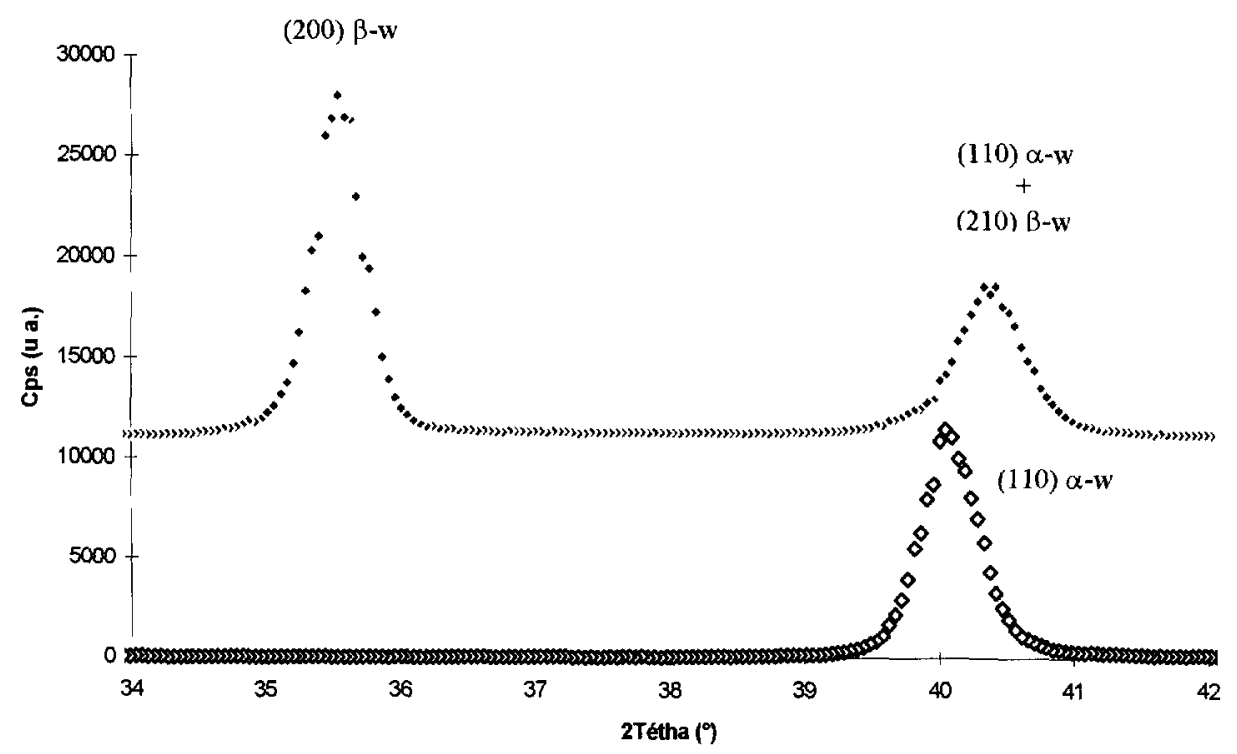

Fig. 2. - Pic de diffraction (110) de la phase $\alpha$-W ansi que (200) et (210) de la phase $\beta$-W pour un échantillon sans $\beta-\mathrm{W}$ (en bas sur la figure) et pour un échantıllon contenant du $\beta-\mathrm{W}$ (en haut sur la figure)

[(110) diffraction pic of the $\alpha$-W phase and (200) and (210) ones of the $\beta$-W phase concerning a sample without $\beta$-W phase (lower part of the figure) and a sample with $\beta$-W (upper part of the figure).]

expliquer la variation des contraintes observée en raison de leur faible variation en fonction des paramètres de cette étude.

L'analyse par diffraction des rayons $\mathrm{X}$ a révélée, pour certains films, la présence de pics de diffraction supplémentaires par rapport à la structure cubique centrée du tungstène massif (phase $\alpha-W$ ). Ces pics ont une position proche de ceux de la structure A-15 de la phase $\beta-W$. La figure 2 montre les pics de diffraction d'un dépôt ne contenant pas de $\beta$-W et d'un échantillon contenant une forte proportion de $\beta$-W (pics (110) $\alpha-W,(200) \beta$-W et (210) $\beta$-W). Nous avons déterminé les proportions des phases $\alpha-W$ et $\beta-W$ pour chaque échantillon.

Sachant que pour un dépôt polycristallin, dont les domaines cohérents de diffraction sont orientés au hasard, l'intensité intégrée d'un pic de diffraction ( $h k l$ ) est proportionnelle au pouvoir de diffraction $P^{\prime}$ provenant des domaines cohérents de volume total $V_{\imath}$, ayant leurs plans $(h k l)$ en coïncidence de Bragg. $P^{\prime}$ s'écrit selon Warren [20] :

$$
P^{\prime}=K\left(1 / v_{\imath}\right)^{2} V_{\imath} \lambda^{3} M_{\imath}\left(h, k, l,\left.|| F_{\imath}(h, k, l)\right|^{2}\right. \text { L.P. }
$$

où $i=\alpha$ ou $i=\beta V_{\imath}$ est le volume diffractant de chaque phase, $v_{\imath}$ le volume de chaque maille élémentaire, $M_{\imath}(h, k, l)$ le facteur de multiplicité, $F_{2}(h, k, l)$ le facteur de structure. L.P. est le facteur de Lorentz Polarisation, $\lambda$ la longueur d'onde des rayons X utilisés $(0,15406 \mathrm{~nm})$ et $K$ une constante.

On estime $V_{\alpha}$ et $V_{\beta}$, à partir des données expérimentales et de la relation précédente. En appliquant la formule suivante,

$$
\% \beta-W=\frac{V_{\beta}}{V_{\alpha}+V_{\beta}}
$$

où $V_{\alpha}$ est le volume diffractant de la phase a et $V_{\beta}$ est celui de la phase $\beta$, on détermine le 


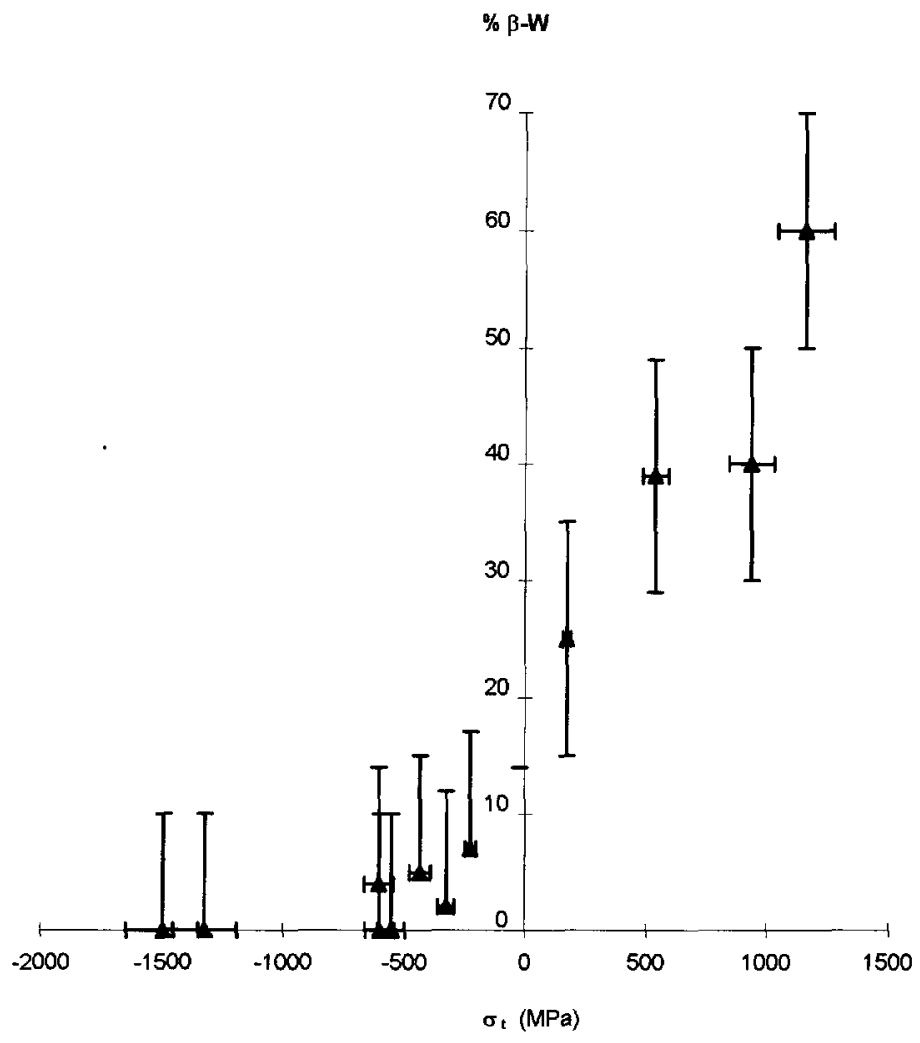

Fig 3. - Pourcentage de $\beta-W$ en fonction de la contrainte totale dans le film.

[ $\beta$-W\% phase versus total stress in the film.]

pourcentage de phase $\beta$-W dans chaque échantillon. Toutefois, il ne représente qu'une approximation et ne permet qu'une étude relative.

Le pourcentage de $\beta$-W représenté en fonction de la contrainte totale dans le film (Fig. 3) montre que la présence importante de phase $\beta$-W est étroitement liée à un état de tension des films. Les dépôts en compression ne possèdent que pas ou très peu de $\beta$-W $(<10 \%)$ alors que le pourcentage de $\beta$-W, pour les films en tension, peut atteindre de très fortes proportions $(>60 \%)$. On peut penser que pour minimiser l'énergie élastique emmagasinée lors d'un dépôt réalisé avec une faible puissance rf (films tendus), la formation de la phase $\beta$-W est privilégiée par rapport à celle de la phase $\alpha-W$, du fait de son volume atomique plus important. Ceci peut expliquer que les films en forte compression ne possèdent pas ou très peu de $\beta$-W et que plus les contraintes de tension augmentent, plus la phase $\beta$-W peut prendre une part importante dans le film. Ce résultat a déjà été observé par Pétroff [21] dans des dépôts de tungstène également obtenus par pulvérisation magnétron. Il explique également que plus la puissance est importante, plus la température du substrat est élevée ; ce qui favorise une transition de phase de $\beta$-W vers $\alpha$-W. Le pourcentage de phase $\beta$-W diminue donc naturellement quand la puissance rf augmente.

Malgré la différence fondamentale entre les deux méthodes, il est parfois possible de comparer les valeurs des contraintes obtenues par diffraction des rayons $\mathrm{X}$ (intra-grannulaires) avec celles 


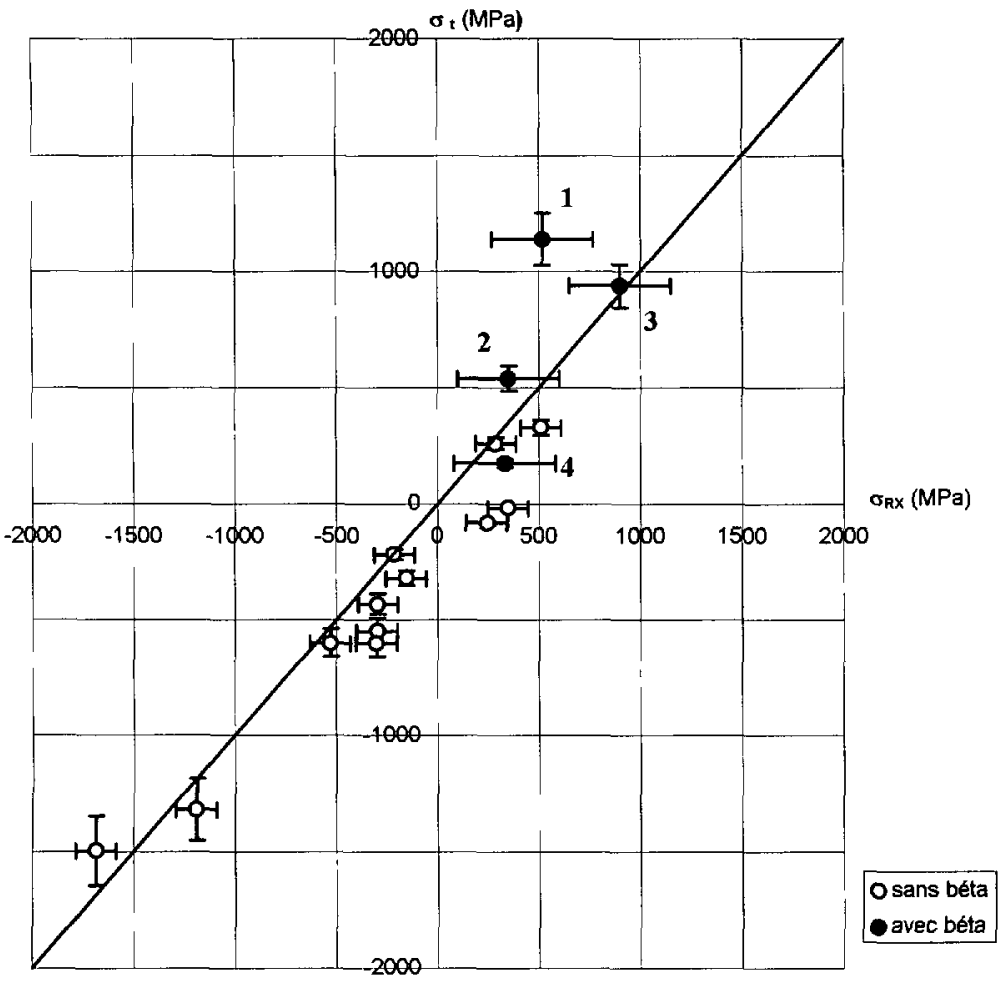

Fig. 4 - Comparaison des contraintes totales par la méthode de la flèche (L.2.M.) et des contraintes calculées à partır des contraintes dans les deux phases $\left(\sin ^{2} \psi\right)$. Les cercles vides représentent les échantillons sans $\beta$-W et ceux pleins, les échantillons avec $\beta-\mathrm{W}$

[Total stress (determined by the curvature method) and stress calculated from the stress in each $W$ phase. The empty circles represent the samples without $\beta-W$ phase and full ones represent samples with $\beta$-W.]

obtenues par la méthode de la flèche (contraintes globales). Pour les dépôts comprimés (donc quasiment monophasés), les contraintes totales peuvent être supposées égales à celles présentes dans la phase $\alpha-\mathrm{W}$ en supposant que les contributions à la contrainte totale de la phase $\beta$-W, des zones amorphes, des joints de grains, ou encore des amas de défauts sont faibles.

Pour les films où la phase $\beta$-W est fortement présente, une estimation, à partir de la détermination des contraintes dans chaque phase, des contraintes totales du matériau biphasé est possible. Après avoir mesuré les contraintes dans les deux phases nous avons appliqué la règle des mélanges En première approximation, elle s'écrit [11] :

$$
\sigma_{\text {calculée }}=\frac{V_{\alpha} \sigma_{\alpha}+V_{\beta} \sigma_{\beta}}{V_{\alpha}+V_{\beta}}
$$

où $\sigma_{\alpha}$ et $\sigma_{\beta}$ sont respectivement les contraintes dans les phases $\alpha$-W et $\beta$-W, $V_{\alpha}$ et $V_{\beta}$ les volumes diffractants de chacune des phases. Les résultats de cette estimation sont regroupés dans le tableau I. On remarque que les valeurs des contraintes résiduelles dans les deux phases sont parfors très différentes. Par exemple, l'échantillon $\mathbf{n}^{\circ} 2$ ne possède pratiquement pas de contrainte dans la phase $\alpha$-W, alors qu'il est soumis à des contraintes de tension de $+1 \pm 0,3 \mathrm{GPa}$ dans la phase $\beta$-W. 
Tableau I. - Comparaıson entre la contrainte totale présente dans les dépôts bıphasés et la contrainte calculée selon la règle des mélanges.

[Total stress in biphased samples and stress calculated from the "règle des mélanges".]

\begin{tabular}{|c|c|c|c|c|c|}
\hline Ech. & $\begin{array}{c}\sigma_{\alpha} \\
(\mathrm{MPa})\end{array}$ & $\begin{array}{c}\sigma_{\beta} \\
(\mathrm{MPa})\end{array}$ & $\% \beta$ & $\begin{array}{c}\sigma_{\text {calculée }}(\mathrm{MPa}) \\
\text { rayons X }\end{array}$ & $\begin{array}{c}\sigma_{\mathrm{t}}(\mathrm{MPa}) \\
\text { flèche }\end{array}$ \\
\hline 1 & $-180 \pm 90$ & $980 \pm 260$ & $60 \pm 10$ & $516 \pm 300$ & $1160 \pm 120$ \\
\hline 2 & $-47 \pm 25$ & $940 \pm 255$ & $40 \pm 10$ & $348 \pm 240$ & $539 \pm 54$ \\
\hline 3 & $923 \pm 250$ & $870 \pm 190$ & $40 \pm 10$ & $902 \pm 214$ & $937 \pm 94$ \\
\hline 4 & $220 \pm 100$ & $670 \pm 155$ & $25 \pm 10$ & $332 \pm 160$ & $175 \pm 18$ \\
\hline
\end{tabular}

On note sur la figure 4 que les contraintes totales estimées à partir de la diffraction des rayons $\mathrm{X}$, conduisent à des valeurs comparables à celles données par la méthode de la fièche. Les points expérimentaux (sauf le $\mathrm{n}^{\circ} 1$ ) sont très proches de la droite $y=x$, qui représente l'égalité parfaite entre les contraintes déterminées à partir des deux méthodologies. Cette comparaison entre les contraintes totales, calculées à partir des contraintes dans les deux phases, et celles déterminées expérimentalement par la méthode de la flèche, nous apporte des informations très intéressantes. Cette cohérence des résultats indique que les constantes élastiques des films minces étudiés semblent très voisines de celles du tungstène massif et que les effets dus aux joints de grains, souvent très importants dans les films minces polycristallins, sont ici relativement faibles. Ce résultat est à comparer à ceux d'une étude précédente [9], sur des films de $100 \mathrm{~nm}$ de W obtenus par pulvérisation directe par un faisceau d'argon, qui a montré de gros écarts entre les valeurs des contraintes mesurées par les deux méthodes (-2,9 GPa par flèche et -5,2 GPa par la méthode des " $\sin ^{2} \psi$ " pour un dépôt brut d'élaboration). Cet écart a été attribué à la forte présence de joints de grains ( $25 \%$ du volume global, ces joints de grains étant considérés comme une seconde phase au sens mécanique), ou à une variation importante (40\%) du module d'Young des films.

L'interprétation des résultats, obtenus pour le dépôt $n^{\circ} 1$, reste cependant plus complexe. En effet, les contraintes calculées sont beaucoup plus faibles que celles déterminées par interférométrie $(0,5 \mathrm{GPa}$ au lieu de 1,2 GPa). On peut attribuer cet écart à une plus grande sensibilité aux proportions de phases (connues approximatıvement à partir de la formule (6)) utilisées lors du calcul, les contraintes étant très différentes dans les deux phases (faible dans la phase $\alpha$-W et forte dans la phase $\beta-W$ ). D'autre part, la méconnaissance des constantes élastiques de la phase $\beta$-W est également une source d'erreur non négligeable pour la détermination des contraintes dans cette phase puisque nous avons utilisé les constantes élastiques du tungstène massif (phase $\alpha-W$ ) qui peuvent être différentes de celles de la phase $\beta-W$. De plus, l'hypothèse d'isotropie élastique, qui est indispensable pour l'utilisation de la méthode des " $\sin ^{2} \psi$ ", n'est très certainement pas réalisée dans la phase $\beta$-W.

En résumé, bien que le problème des contraintes résiduelles dans les matériaux polyphasés soit un sujet délicat et non encore entièrement résolu dans les matériaux massifs $[10,11]$, 1 est clair que pour les films où la proportion de phase $\beta$-W est importante, l'on ne puisse pas considérer uniquement les contraintes dans la phase $\alpha$-W comme étant représentatives des contraintes totales présentes dans le film. L'analyse des contraintes dans la phase $\beta-W$ est donc indispensable pour une meilleur compréhension de l'état mécanique et structural du film. Cependant, il semble nécessaire au préalable, de déterminer avec précision les valeurs des constantes élastiques de cette phase 


\section{Microstructure intra-granulaire de la phase $\alpha-W$}

Dans cette seconde partie, nous allons analyser uniquement la structure intra-granulaire de la phase $\alpha-W$ pour tous les films étudiés. Pour survre l'évolution de la structure intra-granulaire de la phase $\alpha$-W des films de tungstène en fonction des différentes conditions de dépôt, nous avons déterminé par la méthode des $\sin ^{2} \psi$, le paramètre de maille libre de contrainte, $a_{0}$. Nous avons reporté, sur la figure 5 , les valeur de $a_{0}$ en fonction des contraintes intra-granulaires déterminées par diffraction des rayons X dans la phase $\alpha$-W. On remarque une chute du $a_{0}$ quand on va de la compression à la traction : en effet, $a_{0}$ passe de $0,3173 \mathrm{~nm}$ pour un dépôt ayant une contrainte de compression de $-1,7 \pm 0,2 \mathrm{GPa}$ à $0,3164 \mathrm{~nm}$ pour un dépôt présentant une contrainte de $+0,9 \pm 0,2 \mathrm{GPa}$ en tension. L'erreur sur les valeurs du paramètre de maille libre de contrainte est de l'ordre de $0,0001 \mathrm{~nm}$, ce qui est très largement inférieur à l'amplitude de l'évolution observée. Le paramètre de maille libre de contrainte, pour les dépôts en compression, est très fortement supérieur à celui du tungstène massif $\alpha-W(0,3165 \mathrm{~nm})$. Toutefois, des variations du paramètre cristallin deux fois plus importantes ont déjà été rapportées dans des films de $W$ de $100 \mathrm{~nm}$ d'épaisseur déposés par pulvérisation [9]. Cette forte valeur du paramètre crıstallin libre des contraintes traduit une présence importante de facteurs augmentant le volume de la maille cristalline : défauts ponctuels de type "interstitiel", impuretés de volume atomıque supérıeur à celui de l'atome de W, etc. À l'opposé, les films en tension possèdent un paramètre de maille légèrement inférieur à celui du tungstène massif, indiquant une structure intra-granulaire faiblement lacunaire.

Les microdéformations, qui représentent l'écart quadratique moyen des déformations à l'échelle nanométrique, traduisent l'écart à la perfection cristalline d'un cristal réel Au même tıtre que le paramètre de maille libre de contrainte, les microdéformations sont le reflet de la présence d'impuretés, de défauts ponctuels et de dislocations. Pour les échantillons qui possèdent du $\beta$-W, l'analyse par la méthode de la largeur intégrale n'a pu être réalisée. En effet, les pics de diffraction (110) et (220) de la phase $\alpha-W$, sont superposés aux pics (210) et (420) de la phase $\beta-W$. Ainsi, l'analyse des microdéformations n'a aucun sens physique. En revanche, pour les dépôts ne contenant pas de $\beta$-W, l'analyse conduit à de fortes valeurs de microdéformations $(\approx 0,4 \%)$. Ces valeurs sont 10 fois supérieures à celles d'un matériau massif laminé à $70 \%$. Ceci a déjà été observé à diverses reprises $[7,9]$ dans des films minces et confirme la présence de nombreux défauts ponctuels à l'intérieur même de la maille cristalline des films en compression. La diminution des microdéformations, avec les contraintes de compression présentes dans la phase $\alpha$-W des films, est de très faible amplitude mais semble confirmer l'évolution du paramètre cristallin, à savoir le rapprochement de l'état structural du film vers la perfection cristalline (moins de défauts ponctuels dans la malle).

La méthode de la largeur intégrale donne, outre les microdistorsions du réseau cristallin, la taille des domaines cohérents de diffraction. Elle est de l'ordre de $50 \mathrm{~nm}$ pour tous nos dépôts. Deux modèles de genèse des contraintes résiduelles intrinsèques dans les films minces sont couramment présentés dans la littérature. Le premier, proposé par Finegan et Hoffmann [22, 23], est le modèle de relaxatıon aux joints de grain, dit modèle "G.B." (Grain Boundary). Le mécanisme de ce modèle met en jeu des particules de faible énergie. Les premiers stade de croissance du film sont caractérisés par la présence d'îlots. La coalescence de ces derniers, dans le but de minimiser les porosités inter-granulaires, est à l'origine des contraintes de tension dans le film. Ce modèle est cohérent avec les valeurs du paramètre de maille libre de contraintes dans la phase $\alpha$-W inférieures à celle du tungstène massif que nous avons rapporté dans cet article pour les films en tension. Ces valeurs du paramètre de maille traduisent bien une structure à tendance lacunaire. Selon le modèle G.B., les contraintes en tension dans les films sont inversement proportionnelles à la taille des domaines cohérents de diffraction. En conséquence, 


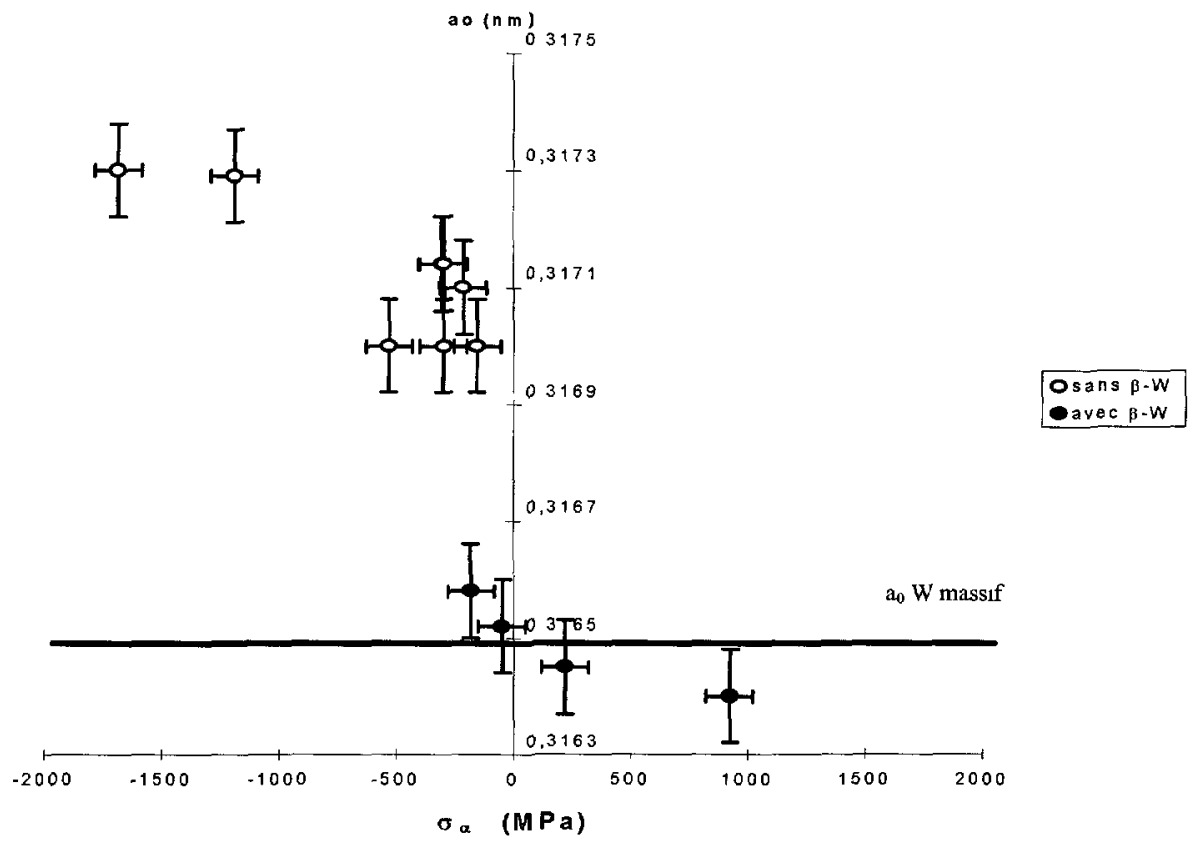

Fig. 5. - Paramètre de malle libre de contrainte $\left(a_{0}\right)$ en fonction de la contrainte détermınée par diffraction des rayons $X$. Les cercles vides représentent les dépôts sans $\beta$-W et ceux pleins, les échantillons avec $\beta-\mathrm{W}$. La droite horizontale représente la valeur du paramètre lıbre de contrainte du tungstène massif.

[Free stress lattice parameter versus stress determined by XRD in the $\alpha$-W phase. The empty circles represent the samples without $\beta$-W phase and full ones represent samples with $\beta$-W. The straight line represents the bulk $W$ free lattice parameter.]

la structure intra-granulaire des films est faiblement influente sur leur état mécanıque. Ceci semble être confirmé par le paramètre de maille (proche de celui du W massif) qui n'évolue pas ou peu en fonction de l'amplitude de la contrainte de tension observée. Nous avons vérifié ces deux points lors d'une étude sur des films minces de platine de $150 \mathrm{~nm}$ déposés à faible énergie [24].

Le second modèle, "l'atomic peening", a été développé par d'Heurle [25]. Il suggère que les contraintes de compression observées dans les films déposés par pulvérisation sont le résultat du bombardement du film par des particules de forte énergie. Celul-ci induit une densification du film et ainsi, une augmentation du volume du dépôt. Cette augmentation de volume, contrariée par la liaison film-substrat, donne naissance à des contraintes de compression dans le plan du film.

Lors d'un dépôt par pulvérisation, les deux mécanismes de genèse des contraintes sont en concurrence. La variation d'un paramètre de dépôt, en particulier la puissance rf, produit de grandes variations sur le rapport "particules de forte énergie/particules de faible énergie" [26]. Il en résulte une transition de la tension vers la compression quand ce rapport augmente, c'est-à-dire quand la puissance rf augmente.

L'origine des contraintes en compression dans le film est donc étroitement liée à une densification à l'échelle atomique du film pendant sa croissance. Ce bombardement conduit à des 
modifications à l'intérieur même de la maille cristalline dont le paramètre de maille libre de contrainte est perturbé. Plus la puissance rf fournie lors du dépôt est importante, plus les particules, qui viennent se déposer sur le substrat, sont énergétiques. Ceci augmente l'effet de bombardement que subit le film déjà déposé et génère une structure de plus en plus dense et comprimée et ainsi un paramètre $a_{0}$ de plus en plus grand. Une corrélation étroite entre $a_{0}$ et les contraintes de compression dans la phase $\alpha-W$ a été rapportée dans des films minces de tungstène déposés par pulvérisation [27]. Une décroissance du paramètre de maille libre de contrainte jusqu'à la valeur du tungstène massif a été mise en évidence lors d'une relaxation des contraintes de compression induite par irradiation par des ions $\mathrm{Ar}^{+}$à haute énergie $(340 \mathrm{keV})$. À partir de l'ensemble de ces données expérimentales intra-granulaires, on peut relier quantitativement, l'augmentation du paramètre de maille libre de contrainte à celle des contraintes de compression. Si l'on considère le paramètre cristallin ( $a_{0}$ tendu $)$ des films soumis à des contraintes de tension dans la phase $\alpha$-W comme référence, on peut estimer les contraintes induites par l'augmentation du paramètre de maille libre de contrainte par la formule suivante [28] :

$$
\sigma=\frac{-E}{1-\nu} \ln \left(\frac{a_{0 \max }}{a_{0 \text { tendu }}}\right)
$$

où $a_{0}$ max représente la valeur maximum du paramètre cristallin. On trouve une contrainte de compression de $-1,5 \mathrm{GPa}$ qui est cohérente avec les valeurs de contraintes en compression observées lors de cette étude. Ceci confirme le rôle essentiel de l'atomic peening sur la nature de la microstructure intra-granulaire et sur les contraintes pour des dépôts en compression.

Ce lien entre microstructure intra-granulaire et contraintes de compression nous semble très important car beaucoup plus intimement lié au processus de densification que la morphologie proprement dite du film. En effet, bien que celle-ci soit également fortement influencée par le bombardement de densification, elle ne représente qu'une image à l'échelle microscopique d'effets se produisant à l'échelle nanométrique. Ce point confirme totalement les conclusions des études précédentes sur ce sujet $[1,2]$. La mise en évidence de variations de la morphologie des films était spectaculaire pour de grandes varrations de puissance ou de température, mars l'étude de la zone de transition brutale de la tension vers la compression n'avait apportée que peu d'informations. L'étude intra-granulaire réalisée par diffraction des rayons $\mathrm{X}$ apporte sans aucun doute une confirmation expérimentale directe et originale des effets du bombardement atomique de densification et constitue une nouvelle preuve du modèle de "l'atomic peening".

\section{Conclusion}

Dans cet article, nous avons montré que la présence de la phase $\beta$-W semble fortement favorisée par un état de tension des films. La méthode des " $\sin ^{2} \psi$ " nous a permis, dans le cas de films polyphasés $(\alpha-W$ et $\beta-W)$, de calculer les contraintes globales sur l'ensemble des domaines diffractants. En les comparant à ceux donnés par la méthode de la flèche, on s'aperçoit que les écarts sont relativement faibles. L'association des résultats donnés par la méthode de la flèche (contraintes globales sur tout le film) avec ceux déterminés à partir de la méthode des " $\sin ^{2} \psi$ " (contraintes dans la phase diffractante et paramètre de maille libre de contrainte), nous a permis de montrer le lien étroit entre la nature et l'amplitude des contraintes présentes dans les films minces et leur microstructure. En effet, nous avons montré que l'état de tension dans les films minces de W n'est pas influencé par sa microstructure intra-granulaire. Dans ce cas le paramètre de maille libre de contrainte est très proche de celui du $W$ massif. Ce résultat est en parfait accord avec le modèle G.B., qui prévoit la formation de contrainte de tension par attraction inter-granulaire. En revanche, selon le modèle de "l'atomic peening", les contraintes 
en compression, présentes dans certains films, sont générées par un mécanisme agissant à l'échelle atomique au sein des domaines diffractants. Nous avons vérifié que l'augmentation du paramètre de maille libre de contrainte, dans les domaines cohérents de diffraction, induite par le bombardement atomique durant le dépôt, est parfaitement corrélée à celle des contraintes de compression. Ces informations structurales intra-granulaires viennent en complément de celles inter-granulaires rapportées lors des études de morphologie des films. Elles constituent une nouvelle confirmation du bombardement de densification en tant que cause de l'apparition de contraintes intrinsèques de compression dans les films minces déposés par pulvérisation.

\section{Bibliographie}

[1] Hoffmann D.W. and Thornton J.A., Thın Sol. Films 171 (1989) 5.

[2] Haghiri-Gosnet A.M., Ladan F.R., Mayeux C. and Launois H., Stress and microstructure in W sputtered thin films, J. Vac. Sci. Tech. A 7 (1989) 2663-2669.

[3] Thornton J.A., J. Vac. Scz. Technol. 12 (1975) 830.

[4] Noyan I.C. and Goldsmith C.C., Adv. X-Ray anal. 34 (1991) 587.

[5] Noyan I.C., Huang T.C. and York B.R., Residual stress/strain analysis in thin film by X-Ray diffraction, critical Rev. in Sol. State and Mat. Sc. 20 (1995) 125-177.

[6] Badawi K.F., Kalhoun C. and Grilhé J., Formalisme rationel de la méthode de détermination des contraintes résiduelles par diffraction des rayons X, J. Phys III France 3 (1993) 1183.

[7] Castex L., Lebrun J.L., Maeder G. et Sprauel J.M., Détermination des contraintes résiduelles par diffraction des rayons X, Pub. Sci. Tech. (ENSAM, Paris 1981).

[8] Ravet M.F., Badawi K.F., Durand N., Lafontaine H., Barnole V. and Haghiri-Gosnet A.M., Intra granular microstructure and stress in sputtered $\mathrm{W}$ thin films, Mat. Res. Soc. Symp. Proc. 306 (1993).

[9] Durand N., Contraintes résiduelles et microstructure dans des films minces de W, Thèse de Doctorat, Université de Poitiers (1994).

[10] Bloquel F., Barralier L., Bourse G. et Dürr J., Détermination des contraintes résiduelles par diffractométrie $\mathrm{X}$ des couches biphasées, à paraître dans $J$. Phys. Colloq. France IV 1996 (congrès ENSAIS Strasbourg 11/95)

[11] Noyan I.C. and Cohen J.B., An X-ray diffraction study of the residual stress-strain distributions in shot-peened two phase brass, Mat. Scr. Ing. 75 (1985) 191-193.

[12] Stoney G.G., The tension of metallic films deposited by electrolysis, Proc. Royal Soc., London A 82 (1909) 172.

[13] Townsend P.H., Barnett D.M. and Brunner T.A., Elastic relationships in layered composite media with approximation for the case of thin films on a thick substrate, J. Appl. Phys. 62 (1987) 4438.

[14] Kalhoun C., Badawi K.F. et Diou A , Incertitude sur l'analyse des contraintes par diffraction des rayons X, Rev. Phys. Appl. 25 (1990) 1225-1238.

[15] Wagner C.N.J. and Aqua E.N., Adv. $X$ ray Analysis 7 (1964) 46.

[16] Williamson et Hall, Acta Met. 1 (1953) 22.

[17] Durand N., Bimbault L., Badawi K.F. et Goudeau Ph., Mesure par diffraction des rayons $\mathrm{X}$ les microdéformations dans des films minces d'or, J. Phys. III France 4 (1994) 1025.

[18] Campbell D.S., Maissel L.I. and Glang R., Handbook of thin film technology (Mc GrawHill Ed., New York, 1970) p. 123. 
[19] Smithells, Metals Ref. Book, sixth Edition (Brandes, 1978).

[20] Warren B.E., X-Ray Diffraction, Dover Publication (INC New York, 1990) p. 49.

[21] Petroff P. and Sheng T.T., Microstructure growth and stresses in tungsten films deposited by rf sputtering, J. Appl. Phys. 44 (1973) 2545-54.

[22] Finegan J.D. and Hoffmann R.W., stress and stress anisotropy in evaporated iron films, J. Appl. Phys. 30 (1959) 597.

[23] Doljack F.A. and Hoffmann R.W., The origin of stress in Ni films, Thin Sol. Fulms 12 (1972) 71.

[24] Branger V., Détermination de l'état mécanique et microstructural de films mince de Pt, rapport de D.E.A. (1994)

[25] D'Heurle F.M., Al films deposited by rf sputtering, Metall. Trans. 1 (1970) 725.

[26] Windischmann H.. Intrinsic stress in sputtered thin films, Crit. Rev. in Sol. State and Mat. Sci. 17 (1992) 547-596.

[27] Durand N., Badawi K.F., Goudeau Ph. et Naudon A., Évolution des contraintes résiduelles dans les films minces de $W$ en fonction de l'irradiation, J. Phys. III France 4 (1994) 25-34.

[28] Durand N., Badawi K.F. and Goudeau Ph., à paraître dans J. Appl. Phys. 